\title{
TOPOLOGY OF COMPLEX POLYNOMIALS VIA POLAR CURVES
}

\author{
Pierrette Cassou-Noguès and Alexandru Dimca
}

\section{The main results}

The use of the local polar varieties in the study of singular spaces is already a classical subject, see Lê-Teissier [LT] and the references therein.

In this note we consider the global polar curves associated with an affine smooth hypersurface $F$ in $\boldsymbol{C}^{n}$. Instead of considering the higher dimensional polar varieties associated with $F$, we choose to look at the polar curves for the various generic linear sections of $F$. This approach is motivated by our use of classical dual varieties and also by our main interest in numerical invariants describing the topology of $F$ in terms of these family of polar curves.

More precisely, let $f \in C\left[x_{1}, \ldots, x_{n}\right]$ be a polynomial and assume that the fiber $F_{t}=f^{-1}(t)$ is smooth and connected. Our main result computes the Euler characteristic $\chi\left(F_{t}\right)$ of the hypersurface $F_{t}$ in terms of the polar invariants of the intersections $F_{t} \cap E^{k}$, where $E^{k}$ is a general linear subspace in $C^{n}$ of codimension $k$, for $k=0,1, \ldots, n-1$.

First we define these polar invariants. For any hyperplane

$$
H: h=0 \text { where } h(x)=h_{0}+h_{1} x_{1}+\cdots+h_{n} x_{n}
$$

we define the corresponding polar variety $\Gamma_{H}$ to be the union of the irreducible components of the variety

$$
\left\{x \in C^{n} \mid \operatorname{rank}(d f(x), d h(x))=1\right\}
$$

which are not contained in the critical set $S(f)=\left\{x \in C^{n} \mid d f(x)=0\right\}$ of $f$.

Note that $\Gamma_{H}$ depends only on the direction $H^{d}=\left(h_{1}: \cdots: h_{n}\right) \in \boldsymbol{P}^{n-1}$ of the hyperplane $H$.

LEMMA 1. For a generic hyperplane $H$ we have the following properties.

(i) The polar variety $\Gamma_{H}$ is either empty or a curve, i.e. each irreducible component of $\Gamma_{H}$ has dimension 1. of $H$.

(ii) $\operatorname{dim}\left(F_{t} \cap \Gamma_{H}\right) \leq 0$ and the intersection multiplicity $\left(F_{t}, \Gamma_{H}\right)$ is independent

(iii) The multiplicity $\left(F_{t}, \Gamma_{H}\right)$ is equal to the number of tangent hyperplanes to $F_{t}$ parallel to the hyperplane $H$. For each such tangent hyperplane $H_{a}$, the

Received July 3, 1998. 
intersection $F_{t} \cap H_{a}$ has precisely one singularity, which is an ordinary double point.

Note that (i) and (ii) above are well-known, see for instance Tibar [Ti]. On the other hand, the last property (iii) is exactly the analog of the defining property of a projective Lefschetz pencil, see [L].

Definition 2. The non-negative integer $\left(F_{t}, \Gamma_{H}\right)$ is called the polar invariant of the hypersurface $F_{t}$ or of the polynomial $f$ at the point $t$, and is denoted by $P\left(F_{t}\right)$ or by $P(f, t)$, depending on the point of view we want to emphasize.

Note that $P\left(F_{t}\right)$ corresponds exactly to the classical notion of class of a projective hypersurface.

Our main result is the following.

THEOREM 3. For a generic hyperplane $H$, the homotopy type of the fiber $F_{t}$ is obtained from the homotopy type of the section $F_{t} \cap H$ by attaching $P\left(F_{t}\right)$ cells of dimension $n-1$.

In particular

$$
P\left(F_{t}\right)=(-1)^{n-1}\left(\chi\left(F_{t}\right)-\chi\left(F_{t} \cap H\right)\right)
$$

In the next section we describe geometrically what is meant by a generic hyperplane is these statements, see Theorem $3^{\prime}$. We note here just that the Zariski open set of hyperplanes $H$ for which Lemma 1 (iii) holds is smaller than the open sets corresponding to the claims in Lemma 1 (i) and (ii) and in Theorem 3 .

Corollary 4.

$$
\chi\left(F_{t}\right)=\sum_{h=0, n-1}(-1)^{n-1-k} P\left(F_{t} \cap E^{k}\right)
$$

where $E^{k}$ is a generic linear subspace in $C^{n}$ of codimension $k$, for $k=0,1, \ldots$, $n-1$.

The last term in this sum $P\left(F_{t} \cap E^{n-1}\right)$ is set by convention to be the degree of the polynomial $f$.

To explicitly compute the polar invariant $P(f, t)$ one may proceed as follows. Let $\left(\gamma_{i}\right)_{i}$ be the finite set of fixed parametrisations, one for each branch at infinity of the polar curve $\Gamma_{H}$. Each such parametrisation is given by a Laurent series (convergent in a punctured disc at the origin)

$$
\gamma(s)=a_{k} s^{k}+a_{k+1} s^{k+1}+\cdots
$$

where $k \in \boldsymbol{Z}, k<0$ and $a_{j} \in \boldsymbol{C}^{n}$ for all $j \geq k$. For such a Laurent series (even for series with $k \geq 0$ ), we set ord $\gamma=k$ if $a_{k} \neq 0$. With this notation we have the following result. 
Proposition 5. (i) $P(f, t)=-\sum_{l} \operatorname{ord}\left(f\left(\gamma_{i}\right)-t\right)$;

(ii) The function $P(f,-)$ takes its maximal value on an open set $U=\boldsymbol{C} \backslash\left\{c_{1}, \ldots, c_{m}\right\}$.

The function $P(f,-)$ has a jump at a value $c \in C$, say $c=c_{J}$, if and only if there is a parametrisation $\gamma^{\prime}$ for a branch at infinity of the polar curve of $F_{c}$ such that $\lim _{s \rightarrow 0} f\left(\gamma^{\prime}(s)\right)=c$. When this is the case, then $P(f, u)-P(f, c)=$ $\sum \operatorname{ord}\left(f\left(\gamma^{\prime}\right)-c\right)$ where the sum is extended to all the parametrisations $\gamma^{\prime}$ with the above property.

Assume now that the polynomial $f$ has only isolated singularities. Then Artal-Bartolo, Luengo-Velasco and Melle-Hernández [ALM] have introduced some (possibly negative) integers $\lambda(f)$ and $\lambda(f, t)$ called Milnor numbers at infinity such that

(i) the Euler characteristic of the generic fiber $F_{\text {gen }}$ of $f$ is given by

$$
\chi\left(F_{\text {gen }}\right)=1+(-1)^{n-1}(\mu(f)+\lambda(f))
$$

where $\mu(f)$ is the total Milnor number of $f$, and

(ii) if $F_{t}$ is any smooth fiber of $f$, then

$$
\chi\left(F_{t}\right)=1+(-1)^{n-1}(\mu(f)+\lambda(f)-\lambda(f, t))
$$

In this case we have the following result.

Proposition 6. (i) For any polynomial $f$ and a generic hyperplane $H$, the critical set of the restriction $f_{H}: H \rightarrow C$ of $f$ to $H$ satisfies $\operatorname{dim} S\left(f_{H}\right) \leq$ $\max (\operatorname{dim} S(f)-1,0)$.

(ii) Assume that $f$ has only isolated singularities. Then for a generic hyperplane $H$, the restriction $f_{H}: H \rightarrow C$ of $f$ to $H$ has only isolated singularities and

$$
P(f, t)=\mu(f)+\mu\left(f_{H}\right)+\lambda(f)+\lambda\left(f_{H}\right)-\lambda(f, t)-\lambda\left(f_{H}, t\right)
$$

In particular, for all $t \in C$ one has

$$
\lambda(f, t)+\lambda\left(f_{H}, t\right) \geq 0
$$

Remark 7. (i) When $\operatorname{dim} S(f) \leq 0$, it is not true in general that $\operatorname{dim} S(f)>\operatorname{dim} S\left(f_{H}\right)$ for a generic hyperplane. To see this, it is enough to consider the polynomial $f=x_{1}^{d}+\cdots+x_{n}^{d}$ for which $\operatorname{dim} S(f)=\operatorname{dim} S\left(f_{H}\right)=0$ or the polynomial in our Example 11 below where $S(f)=\emptyset$ and $\operatorname{dim} S\left(f_{H}\right)=0$.

(ii) When $f$ has isolated singularities on $C^{n}$ and at infinity (in the sense that the projective closure of $F_{t}$ has only isolated singularities), then for a generic hyperplane $H$ the restriction $f_{H}$ has no singularities at infinity, in particular $\lambda\left(f_{H}\right)=0$.

It is likely that a similar property holds for the polynomials with isolated singularities at infinity relative to an arbitrary compactification of $C^{n}$, see [ST] for more on this class of polynomials and also Note 3.8 in [Ti].

Moreover, if $f$ and $f_{H}$ are such polynomials, then by our Proposition 6 (ii) 
the function $P(f,-)$ is constant on a neighborhood $U$ of $t_{0}$ if and only if $f$ and $f_{H}$ are locally trivial over $U$.

A general discussion on polar curves from the point of view of their relations to topological triviality at infinity can be found in [Ti], see our Acknowledgements (ii) at the end of this paper. Some results for $n=2$ can be found in Assi $[\mathrm{A}]$.

\section{Proofs, examples and some further results}

Proof of Lemma 1 (i). Let $\phi: \boldsymbol{C}^{n} \rightarrow \boldsymbol{C}^{n}$ be the gradient map associated to $f$. Then there are algebraic subsets $Y_{J}$ in $C^{n}$ for $j=0,1, \ldots, n$ such that

(i) $\operatorname{codim} Y_{j} \geq j$ and

(ii) $\operatorname{dim} \phi^{-1}(y) \geq j$ implies that $y \in Y_{j}$.

If we choose a line $L: y_{1}-h_{1} y_{n}=\cdots=y_{n-1}-h_{n-1} y_{n}=0$ such that $L \cap Y_{J} \subset\{0\}$ for all $j>1$ and that $L$ is not contained in $Y_{1}$, then for the corresponding hyperplane $H: h_{1} x_{1}+\cdots+h_{n-1} x_{n-1}+x_{n}=0$ the variety $\Gamma_{H}=$ closure $\left(\phi^{-1}(L \backslash\{0\})\right.$ is a curve.

This curve has no isolated points as components, since such a point would be defined locally by $(n-1)$ equations, a contradiction.

Proof of Lemma 1 (ii), (iii) and of Theorem 3. This proof is based on the results of Némethi on the Lefschetz theory for affine varieties, see [N1] and, for more details, [N2].

To simplify the notation, in this proof we omit the subscript $t$, e.g. we write $F$ instead of $F_{t}$.

Let $V$ be the projective closure of $F$ in $\boldsymbol{P}^{n}$. Let $H_{\infty}: x_{0}=0$ be the hyperplane at infinity in this projective space $\boldsymbol{P}^{n}$.

In the dual projective space $\hat{\boldsymbol{P}}^{n}$ we use $\left(h_{0}: \cdots: h_{1}\right)$ as homogeneous coordinates. Let $\hat{V} \subset \hat{\boldsymbol{P}}^{n}$ be the dual of the hypersurface $V$.

We introduce the following sets to describe the bad directions of hyperplanes in $C^{n}$. The affine hyperplanes in $C^{n}$ are parametrized by the open set $A=\hat{\boldsymbol{P}}^{n} \backslash\{\infty\}$, where $\infty=(1: 0: \cdots: 0)$ is the point corresponding to $H_{\infty}$. Consider the projection $p: A \rightarrow \boldsymbol{P}^{n-1}$ associating to a hyperplane $H$ its direction $H^{d}$. Note that the fibers of $p$ are precisely the pencils of parallel hyperplanes in $C^{n}$. We define $D(F)=\left\{(x, H) \in F \times A \mid T_{x} F=H\right\}$ and $\hat{F}=\operatorname{pr}_{2}(D(F))$, the dual of the affine variety $F$. Let $D(V)$ be the closure of $D(F)$ in $\boldsymbol{P}^{n} \times \hat{\boldsymbol{P}}^{n}$ and

$$
D(V)_{\infty}=\left\{(x, H) \in D(V) \mid x_{0}=0\right\}
$$

Next let $\hat{V}_{\infty}=\operatorname{pr}_{2}\left(D(V)_{\infty}\right), W_{1}=p\left(\hat{V}_{\infty} \backslash\{\infty\}\right)$.

Let $C=C_{\infty}(\hat{V})$ be the projective tangent cone to the dual variety at the point $\infty$ and let $W_{2}=p(C \backslash\{\infty\})$. When $\infty \notin \hat{V}$, we set $C=W_{2}=\emptyset$. 
EXAMPLE 8. The case $(n=2)$. In this case it is very easy to describe the sets $W_{1}$ and $W_{2}$.

Note first that the directions $H^{d}$ in $\boldsymbol{P}^{1}$ can be identified to the points on the line at infinity $H_{\infty}$, i.e. a direction $(a: b)$ corresponds to the point $(0: b:-a)$.

Under this identification, the directions in $W_{1}$ (resp. $W_{2}$ ) correspond to the points $p \in V \cap H_{\infty}$ such that the germ $(V, p)$ has a tangent direction different from (resp. equal to) $H_{\infty}$.

Moreover, in this case we have the equivalences: $\operatorname{dim}(\hat{V})<1 \Leftrightarrow \operatorname{dim} \phi\left(C^{2}\right)<$ $2 \Leftrightarrow \operatorname{Hess}(f)=0 \Leftrightarrow f$ is a linear form $\Leftrightarrow$ the generic fiber of $f$ has no tangents parallel to a generic direction (note that $\hat{V}$ cannot be a line!).

If we take any polynomial $f \in \boldsymbol{C}[x, y]$, we know that $f=g(h)$ where $g \in \boldsymbol{C}[t]$ and $h \in C[x, y]$ is such that the generic fiber $F_{h}$ of $h$ is connected. It follows that the generic fiber of $f$ is the disjoint union of $k=\operatorname{deg}(g)$ copies of $F_{h}$ and hence we have $\operatorname{Hess}(f)=0 \Leftrightarrow \operatorname{dim} \phi\left(C^{2}\right)<2 \Leftrightarrow$ there is a linear coordinate change of $C^{2}$ such that $f(x, y)=g(x)$.

Note that for $f$ homogeneous, this last statement is a well known fact in classical invariant theory, see [GY], p. 235.

To come back to the proofs, we know by [N1], [N2] that $\operatorname{dim} W_{l} \leq n-2$ for $i=1,2$. We will show the following more precise version of Lemma 1 (ii) and Theorem 3.

THEOREM $3^{\prime}$. For a hyperplane $H$ whose direction $H^{d}$ is not in $W_{1} \cup W_{2}$ the claims of Lemma 1 (ii) and Theorem 3 hold.

First assume that there is a component $D$ in $\Gamma_{H} \cap F$ with $\operatorname{dim} D>0$. At the points of $D$, the tangent hyperplane $T_{x} F$ has always the fixed direction $H^{d}$. But such $a D$ is unbounded and this implies that $H^{d} \in W_{1}$, a contradiction.

Note that the set $U$ of directions $H^{d}$ in $P^{n-1} \backslash\left(W_{1} \cup W_{2}\right)$ for which the corresponding projective pencil $L_{1}^{c}=\operatorname{closure}\left(p^{-1}\left(H^{d}\right)\right)$ is transverse to the dual hypersurface $\hat{V}$ is open and dense (for the exact meaning of transverse in this context, see the discussion of the Cases 1 and 2 below).

It follows that for any direction $H^{d} \in P^{n-1} \backslash\left(W_{1} \cup W_{2}\right)$ we can find a small 1-parameter deformation $H_{s}^{d}$ such that $H_{0}^{d}=H^{d}$ and $H_{s}^{d} \in U$ for all $s \in(0, \varepsilon)$.

Let $\Gamma_{s}$ be the polar variety of $F$ corresponding to the direction $H_{s}^{d}$. Note that all the intersections $\Gamma_{s} \cap F$ are finite, by the first part in Lemma 1 (ii), that was proved above.

We will show below that $\left(\Gamma_{s}, F\right)=\left|\Gamma_{s} \cap F\right|$ for $s \neq 0$. The only way in which $\left(\Gamma_{0}, F\right)$ can have a different value is when some point in the intersection $\Gamma_{s} \cap F$ tend to infinity when $s$ tends to 0 . But this would imply that $H^{d}=H_{0}^{d} \in W_{1}$, a contradiction.

It follows that $\left(\Gamma_{0}, F\right)=\left(\Gamma_{s}, F\right)$ for $s \neq 0$, and therefore in computing this polar invariant we may assume that the pencil $L_{1}^{c}$ is transversal to $\hat{V}$.

To continue the proof, we note that there are two different cases to discuss. 
CASE 1. $\operatorname{deg}(\hat{V})=0 \Leftrightarrow \operatorname{dim}(\hat{V})<n-1 \Leftrightarrow \operatorname{dim} \phi\left(\boldsymbol{C}^{n}\right)<n$.

In this case we can choose $H^{d}$ such that the associated affine pencil $L=p^{-1}\left(H^{d}\right)$ is disjoint from $\hat{V}$. It follows then from [N1], Theorem 2 that this pencil induces a regular function $g: F \rightarrow C$ whose fibers are precisely the hyperplane sections of $F$ by the hyperplanes in $L$ and which is a locally trivial fibration. In particular, the inclusion of $F \cap H$ in $F$ is a homotopy equivalence for any $H \in L$.

In this case it is also clear that $\Gamma_{H} \cap F=\emptyset$, i.e. $P(F)=0$.

CASE 2. $\operatorname{deg}(\hat{V})>0 \Leftrightarrow \operatorname{dim}(\hat{V})=n-1 \Leftrightarrow \operatorname{dim} \phi\left(\boldsymbol{C}^{n}\right)=n$.

For $H^{d}$ generic, the corresponding projective pencil $L^{c}$ given by the closure of $L$ will meet the dual $\hat{V}$ at $\infty$ (if $\infty \in \hat{V}$ ) and at some simple points $a_{1}, \ldots, a_{m}$ on $\hat{V}$, all the intersections being transverse. Then we have

$$
\operatorname{deg}(\hat{V})=\left(\hat{V}, L^{c}\right)_{\infty}+\sum_{l}\left(\hat{V}, L^{c}\right)_{a_{i}}=\operatorname{mult}_{\infty} \hat{V}+m
$$

For each $a_{i}$, the corresponding hyperplane $H_{a_{i}}$ is tangent to $V$ at points in $F$, since $H^{d} \notin W_{1}$.

By [L] or [D1], the section $F \cap H_{a_{i}}$, has exactly one singularity, say $b_{i}$, which is an ordinary double point, i.e. $\mu\left(F \cap H_{a_{i}}, b_{i}\right)=1$.

Then it is easy to see that we have $\Gamma_{H} \cap F=\left\{b_{1}, \ldots, b_{m}\right\}$ for any $H \in L$. Moreover, an easy local computation using $f-t$ as a local coordinate at $b_{i}$ shows that $\left(\Gamma_{H}, F\right)_{b_{i}}=1$. Hence $P(F)=m$ is independent of the choice of the generic hyperplane $H$.

Our Theorem 3 now follows from Theorem 9 (a) in [N1] and Remark 5.7 in [N2].

Remark 9. When the dual hypersurface $\hat{V}$ has only isolated singularities $\left\{a_{1}, \ldots, a_{m}\right\}$, then one can use the formula for the degree of $\hat{V}$, see for instance Kleiman $[\mathrm{K}]$, in order to compute the multiplicity mult $\hat{\sigma}_{\infty}$.

$$
\operatorname{deg}(\hat{V})=(d-1)^{n-1} d-\sum_{i} \mu\left(V, a_{i}\right)-\sum_{l} \mu^{n-1}\left(V, a_{i}\right)
$$

where $d$ is the degree of the polynomial $f$ and $\mu^{n-1}(V, a)$ is the Milnor number of a generic local hyperplane section of the singularity $(V, a)$ as in Teissier [T].

When the part at infinity $V_{\infty}$ has also isolated singularities, then we get via a simple computation based on [D2], p. 159 and p. 162

$$
\operatorname{mult}_{\infty} \hat{V}=\sum_{l}\left(\mu\left(V_{\infty}, b_{i}\right)-\mu^{n-1}\left(V, b_{i}\right)\right)
$$

where $\left\{b_{1}, \ldots, b_{m}\right\}=V_{\text {sing }} \cup V_{\infty, \text { sing }}$, i.e. mult $\hat{V}_{\infty}$ is a sum of local contributions measuring how far is the hyperplane $H_{\infty}$ from a generic hyperplane at each of the points $b_{i}$.

In particular, it follows that if we look at the fibers $F_{t}$ of the polynomial $f$, 
the corresponding dual varieties $\hat{V}_{t}$ may have different degrees and different multiplicities at $\infty$ as well.

Proof of Proposition 5. Let $\left(C_{\gamma}, a\right)$ be the branch of the projective closure $C$ of $\Gamma_{H}$ at a point $a \in H_{\infty}$ corresponding to the parametrisation $\gamma$ written down in the previous section.

We can assume that $a=[0: \cdots: 0: 1]$. Then the parametrisation $\gamma$ corresponds to a mapping

$$
s \mapsto\left[s^{-k}: g_{1}(s): \cdots: g_{n-1}(s): 1\right]
$$

where all the function germs $g_{i}$ are holomorphic.

We obviously have $\left(C_{\gamma}, H_{\infty}\right)_{a}=-k$. By taking the sum over all the branches of $C$ at infinity we get $\operatorname{deg} C=\left(C, H_{\infty}\right)=-\sum_{l}$ ord $=\gamma_{i}$.

Let $\tilde{f}\left(x_{0}, \ldots, x_{n}\right)$ be the homogeneisation of the polynomial $f-t$. Then we have $\left(C_{\gamma}, V\right)_{a}=$ ord $\tilde{f}\left(s^{-k}, g_{1}(s), \ldots, 1\right)=-k d+$ ord $\tilde{f}(1, \gamma(s))=-k d+$ ord $f(\gamma)$, where $d$ is the degree of the polynomial $f$. Summing over all the branches of $C$ at infinity, we get $\sum_{a \in C \cap H_{\infty}}(C, V)_{a}=\operatorname{deg} C \operatorname{deg} V+\sum_{l} \operatorname{ord}\left(f\left(\gamma_{i}\right)-t\right)$. By Bezout Theorem, this gives us the first claim in Proposition 5.

The second claim follows from the first, once we notice the following "stability" of a generic hyperplane: For any $t_{0} \in C$ there exist an $\varepsilon>0$ and a hyperplane $H_{t_{0}}$ satisfying the claims in Lemma 1 with respect to all the hypersurfaces $F_{t}$ for $\left|t-t_{0}\right|<\varepsilon$. When $t_{0}=c$, a special value, one should pick such a hyperplane $H_{c}$ and the parametrisations $\gamma^{\prime}$ are associated to the branches at infinity of the polar curve $\Gamma_{H_{c}}$.

Remark 10. In [N1], [N2], A. Nemethi has introduced for any polynomial $f$ a bad set $\Lambda_{f}$ such that $f$ is a locally trivial fibration over $C \backslash \Lambda_{f}$. This set is not in general the minimal one with this property, see $[\mathrm{NZ}]$ for a polynomial $f$ with $\Lambda_{f}=\boldsymbol{C}$.

We conjecture that all the special values $c_{j}$ from Proposition 5 (ii) are contained in this set $\Lambda_{f}$.

A much harder question is to compare the set $C_{f}=\left\{c_{i}, \ldots, c_{m}\right\}$ to the minimal set $B_{f}$ such that $f$ is locally trivial over $C \backslash B_{f}$. For $n=2$, we have $C_{f}=B_{f}$, since in this case $\chi\left(F_{t} \cap H\right)=d$, the degree of the polynomial $f$ and the set $B_{f}$ is detected by the jumps in the Euler number of $F_{t}$ by Hà-Lê [HL].

Proof of Proposition 6. In fact, we have to prove only the first claim (i), since (ii) follows directly from Theorem 3 and Proposition 5 (ii) above.

Choose a point $y \in C^{n}$ such that $y \neq 0$ and $\operatorname{dim} \phi^{-1}(y) \leq 0$ (note that this choice is generic!). Let $H$ be any hyperplane whose direction $H^{d}$ is defined by $y$ and such that $\operatorname{dim}(S(f) \cap H) \leq \operatorname{dim} S(f)-1$. We will show that the corresponding restriction $f_{H}$ satisfies (i).

Let $e=\operatorname{dim} S(f)$ and let $D$ be a component of the singular locus $S\left(f_{H}\right)$ with $\operatorname{dim} D>\max (e-1,0)$. It follows then that $f_{H}$ is constant along $D$, i.e. there is a value $t$ such that $D \subset F_{t}$. This means that at any point $x \in D \backslash F_{t, \text { sing, the }}$ 
tangent hyperplane $T_{x} F_{t}$ has the same direction $H^{d}$. This is a contradiction since

$$
\operatorname{dim}\left(D \cap F_{t, \text { sing }}\right) \leq \operatorname{dim}(S(f) \cap H) \leq e-1
$$

EXAMPLE 11. Consider the polynomial $f=x-3 x^{3} y^{2}+2 x^{4} y^{3}+y z$, which is the simplest of the polynomials $f_{n, q}$ introduced in [PZ], i.e. $n=q=1$.

This polynomial is equivalent to a linear form via an automorphism of $\boldsymbol{C}^{3}$, in particular $S(f)=\emptyset, \mu(f)=0, \lambda(f)=0$.

One can look at the hyperplane $H: a x+b y+z+c=0$ and note that for $a \neq 0$ the restriction $f_{H}(x, y)=f(x, y,-a x-b y-c)$ has isolated singularities at infinity such that $\lambda\left(f_{H}\right)=\lambda\left(f_{H},-a\right)=1$. By Proposition 6 in which we choose the hyperplane $H$ such that $a \neq-t$, we have the following.

$$
P(f, t)=\mu\left(f_{H}\right)+1
$$

The polar curve $\Gamma_{H}$ is given by the equations

$$
1-9 x^{2} y^{2}+8 x^{3} y^{3}-a y=0 \text { and }-6 x^{3} y+6 x^{4} y^{2}+z-b y=0
$$

To compute the parametrisations for the branches at infinity of the polar curve, it is enough to do this for the plane curve given by the first equation. A direct computation using Proposition 5 (i) yields $P(f, t)=8$ for all $t \in C$. This gives in particular $\mu\left(f_{H}\right)=7>0$ as claimed in our Remark 7 (i) above.

Acknowledgements. (i) The first author would like to thank H. V. Hà and A. Ploski for interesting discussions related to the topic of this paper.

The second author is grateful for the financial support of the Australian Research Council, grant A 69530267. [Ti].

(ii) Upon the receipt of our manuscript, M. Tibar has sent us his preprint

Our Theorem 3 is proved there for families of affine hypersurfaces (which is more general) but under the more restrictive hypothesis that all these hypersurfaces have isolated singularities. Note also that in our Theorem $3^{\prime}$ we make precise the meaning of generic hyperplane in this context by using the constructions by Nemethi [N1], [N2].

We also relate the polar invariants to the Milnor numbers at infinity introduced by Artal-Bartolo, Luengo-Velasco and Melle-Hernández, showing that the sum of these invariants for $f$ and for the restriction to a generic hyperplane $f_{H}$ is positive.

The main result in [Ti] is that the constancy of the invariants $P\left(F_{t} \cap E^{k}\right)$ for $k=0,1, \ldots, n-1$ (in the notation of Corollary 4 above) for $t \in U \subset C \backslash f(S(f)$ ) implies that $f$ is smoothly locally trivial over $U$.

Since most of our results as well as our methods of proof are quite different, the interested reader will find useful to compare the two approaches. 


\section{REFERENCES}

[A] A. Assi, Meromorphic plane curves, preprint.

[ALM] E. Artal-Bartolo, I. Luengo-Velasco and A. Melle-Hernández, Milnor number at infinity, topology and Newton boundary of a polynomial function, preprint.

[D1] A. Dimca, Milnor numbers and multiplicities of dual varieties, Rev. Roumaine Math. Pures Appl., 31 (1986), 535-538.

[D2] A. DimCA, Singularities and Topology of Hypersurfaces, Universitext, Springer, 1992.

[GY] J. H. Grace and A. Young, The Algebra of Invariants, Cambridge Univ. Press, 1903.

[HL] H. V HÀ AND D. T. LÊ, Sur la topologie des polynômes complexes, Acta Math. Vietnam., 9 (1984), 21-32.

[K] S. L. Kleiman, The enumerative theory of singularities, Real and Complex Singularities (Oslo 1976), Sijthoff and Noordhoff, 1977, 297-396.

[L] K. LAMOTKE, The topology of complex projective varieties after S. Lefschetz, Topology, 20 (1981), 15-51.

[LT] D. T. LÊ AND B. TeIsSIER, Variétés polarres locales et classes de Chern des variétés singulières, Ann. of Math., 114 (1981), 457-491.

[N1] A. NÉmETHI, Théone de Lefschetz pour les variétés algébriques affines, C. R. Acad. Sc1. Parıs Sér. I Math., 303 (1986), 567-570.

[N2] A. NÉmEthI, Lefschetz theory for complex affine varietes, Rev. Roumaine Math. Pures Appl., 33 (1988), 233-260.

[NZ] A. NÉMETHI AND A. ZAHARIA, On the bifurcation set of a polynomial function and Newton boundary, Publ. Res. Inst. Math. Scl., 26 (1990), 681-689.

[PZ] L. Paunescu and A. Zaharia, On the Lojasiewicz exponent at infinity for polynomial functions, Koda1 Math. J., 20 (1997), 269-274.

[ST] D. SieRsma AND M. Tibar, Singularities at infinity and their vanıshing cycles, Duke Math. J., 80 (1995), 771-783.

[T] B. Teissier, Cycles évanescents, sections planes et conditions de Whitney, Singularités à Cargèse 1972, Astérisque, 7-8 (1973), 285-362.

[Ti] M. TiBar, Global equisingularity of families of affine hypersurfaces, preprint.

Laboratoire de Mathématiques Pures de Bordeaux

UNIVERSITÉ BORDEAUX I

33405 TALENCE CEDEX

FRANCE

E-mail: cassou@math.u-bordeaux.fr

E-mail: dimca@math.u-bordeaux.fr 